SLAC-PUB-8186

July 1999

updated September 2000

\title{
Beam-Cavity Interaction Circuit at W-Band
}

\author{
Marc E. Hill, W. R. Fowkes, X. E. Lin and David H. Whittum \\ Stanford Linear Accelerator Center, Stanford University, Stanford, CA 94309
}

\begin{abstract}
We describe the design, fabrication and bench-study of a mm-wave cavity employed as a relativistic klystron output structure. The OFE copper cavity was prepared by electrodischarge machining and diffusion bonding, cleaned, and tuned to $91.4 \mathrm{GHz}$. Measured cavity characteristics are presented and compared with theory, including quality factor, $Q$, coupling parameter $\beta$, scattering matrix $S_{11}$, and axial electric field profile $E_{z}$. This work provides the basis for understanding of the cavity as a transfer structure.
\end{abstract}

Submitted to IEEE Transcations on Microwave Theory and Techniques

Work supported by the Department of Energy contract DE-AC03-76SF00515 


\title{
Beam-Cavity Interaction Circuit at W-Band
}

\author{
Marc E. Hill, W. R. Fowkes, X.E. Lin and David H. Whittum
}

\begin{abstract}
We describe the design, fabrication and benchstudy of a mm-wave cavity employed as a relativistic klystron output struct ure. The OFE copper cavity was prepared by electro-discharge machining and diffusion bonding, cleaned, and tuned to $91.4 \mathrm{GHz}$. Measured cavity characteristics are presented and compared with theory, including quality factor, $\mathbf{Q}$, coupling parameter $\beta$, scattering matrix $\mathrm{S}_{11}$, and axial electric field profile $\mathbf{E}_{\mathrm{z}}$. This work provides the basis for understanding of the cavity as a transfer structure.
\end{abstract}

\section{INTRODUCTION}

High energy physics requires compact accelerators operating at extreme gradients, in excess of $100 \mathrm{MV} / \mathrm{m}$. Scalings for trapping, pulsed-heating and other high-gradient phenomena favor miniature, short-wavelength structures to hold off the large electric fields required [1]. A critical problem in the practical study of such structures is the absence of a high-power, mm-wave source. Study of high-field phenomena at conventional wavelengths has required the development of high-power klystrons over several decades of research and engineering [2]. For mm-wave accelerators, we are approaching the corresponding learning curve for highpower handling by a different route, employing an existing accelerator to power mm-wave transfer structures, much as in a relativistic klystron. The benefit of such work is development of know-how in miniature structure fabrication, bench-test, and high-field studies in an accelerator beam environment, in parallel with $\mathrm{mm}$-wave klystron development [3].

Here we report theory and measurements for a single miniature cavity with WR10 output, and $680-\mu \mathrm{m}$ diameter beam tube, prepared for excitation by a relativistic electron beam. This work establishes that the $3.3-\mathrm{mm}$ wavelength $(91.4 \mathrm{GHz})$ scale is accessible to modern machining and measurement techniques, and provides the basis for understanding of cavity performance as a transfer structure.

\section{Tolerances}

To appreciate the machining and tuning tolerances involved it is helpful to consult the circuit-equivalent picture of the application, as seen in Fig. 1. A beam bunched at 11.424 GHz passes through the beam tube, exciting the cavity resonance at the 8 th beam harmonic, $91.392 \mathrm{GHz}$. The beam pulse length of $100 \mathrm{~ns}$ is much longer than the loaded fill time of the cavity ( $T_{f} \approx 1.5 \mathrm{~ns}$ ), so that steadystate is reached early in the pulse. In steady-state, the cavity voltage $\tilde{V}_{c}$ may be may be expressed in terms of the

Work supported by U.S. DOE contract DE-AC03-76SF00515.

M.E. Hill is with the Physics Department at Harvard University, Cambridge, MA 02138.

W.R. Fowkes, X.E. Lin and D.H. Whittum are with the Stanford Linear Accelerator Center, Stanford University, Stanford, California 94309 .

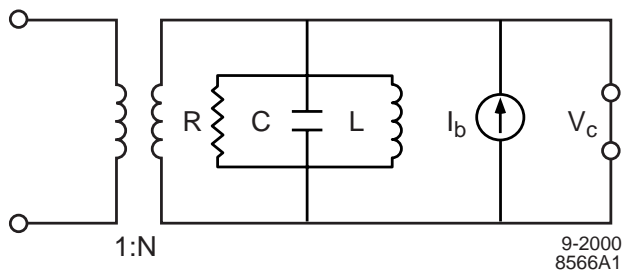

Fig. 1. Circuit equivalent for beam interaction with a single cavity with waveguide output. In the relativistic limit beam-loading admittance is negligible.

beam current $\tilde{I}_{b}$ as [4],

$$
\tilde{V}_{c}=-\frac{1}{2} \cos \psi e^{j \psi} Q_{L}\left[\frac{R}{Q}\right] \tilde{I}_{b},
$$

where the beam-coupling parameter "R-over Q" is defined according to

$$
\left[\frac{R}{Q}\right]=\frac{\left|\tilde{V}_{c}\right|^{2}}{\omega_{0} U},
$$

in terms of stored energy $U$ and resonance angular frequency $\omega_{0}$. The tuning angle $\psi$ is defined in terms of the loaded quality factor, $Q_{L}$, and the drive angular frequency $\omega$, according to

$$
\tan \psi=Q_{L}\left(\frac{\omega_{0}}{\omega}-\frac{\omega}{\omega_{0}}\right) .
$$

Power radiated by the cavity into the guide may then be determined according to

$$
P_{\text {out }}=\frac{\left|\tilde{V}_{c}\right|^{2}}{Q_{e}[R / Q]},
$$

where $Q_{e}$ is the external quality factor. From this one may show that a $10 \%$ reduction in power corresponds to a tuning angle of $\tan \psi=1 / 3$, or cell detuning of $\delta \omega / \omega \approx 1 / 6 Q_{L}$. For a loaded quality factor $Q_{L} \approx 415$,

\section{Fabrichtion and Assembly}

The completed geometry is indicated in Fig. 2. We describe next how this geometry was accomplished, with a set of four nominally identical cavities.

The oxygen-free electronic grade (OFE) copper cavity consists of three layers: a 2.44-mm thick plate with the cavity wire-EDM'd and two featureless plates comprising the cavity top and bottom. (The orientaion of the cavity "top" is at positive $x$ in Fig. 2 and bottom is at negative $x$.) The three layers were diffusion bonded together employing a $1024^{\circ} \mathrm{C}$ heat cycle at 25 psi for 10 minutes. The input waveguide coupling iris was then cut using sinker EDM. Ultrasonic acetone rinse had no measurable effect on wall 


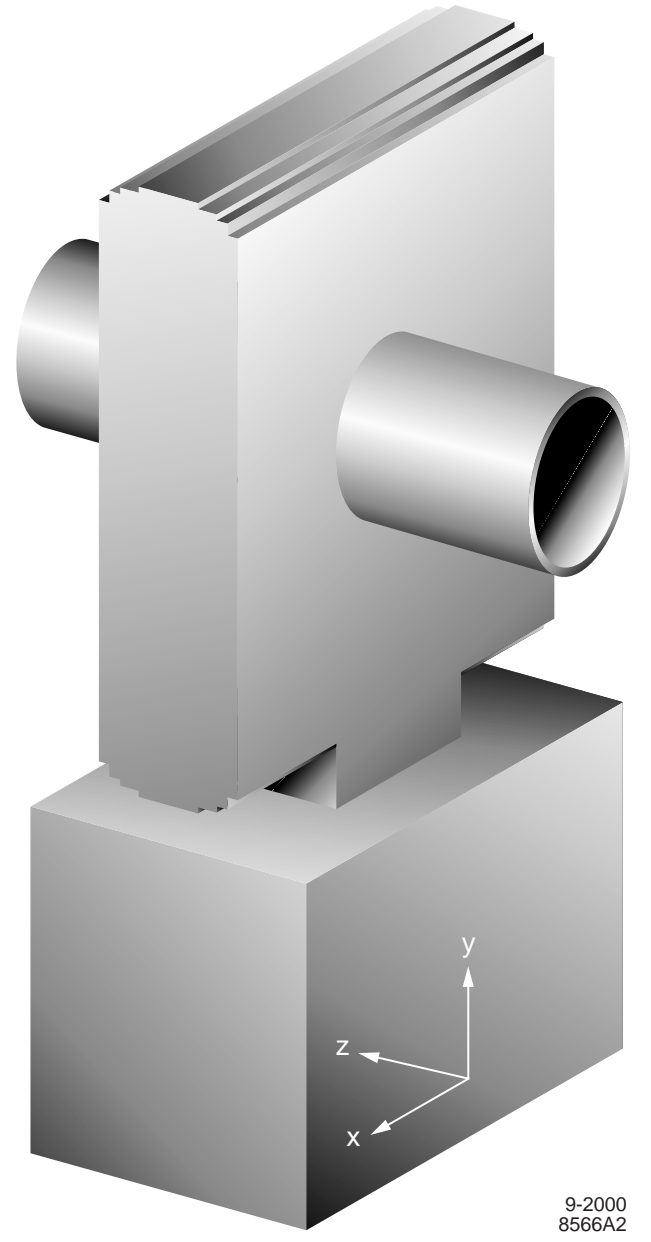

Fig. 2. Geometry of the interior conducting boundaries of the cavity, including coupling iris, beam-tube and WR10 output. The mesh shown is that employed for numerical simulations.

quality factor, and the cavity was subjected to chemical cleaning. This raised the wall $Q$ (prior to cutting of the beam tube) from 1029 to 1252 .

A threaded bolt circle conforming to WR10 0.75" flange was machined into the waveguide opening for each cavity. The width ("a" dimension) of the waveguide openings were intended to match the 0.1 " wide dimension of WR10 guide, but were accidentally machined $0.1 \mathrm{~mm}$ narrower, resulting in a step on each side of the connecting waveguide. Analysis indicated that this would have negligible effect on the measurements.

To cut the beam-tubes, we used sinker EDM for the first, rough cut of the cavity. We then made a final cut of the 340 $\mu \mathrm{m}$ radius using wire EDM. To remove any debris created by the EDM process on the surfaces of the cavity walls, we etched the cavity by pumping an acid solution through the coupling port of the cavity.

\section{Measurements}

To determine the state of the cavity, and as an aid in tuning, we employed a home-built $\mathrm{W}$-band vector network analyzer (VNA) [5]. The VNA permits measurement of the steady-state complex reflection coefficient $\tilde{S}_{11}$ over a range
TABLE I

MEASURED AND THEORETICAL PARAMETERS FOR THE SINGLE CELL CAVITY WITH AND WITHOUT BEAM PORT.

\begin{tabular}{|l|r|r|}
\hline & No Port & Beam Port \\
\hline$Q_{w}$ (analytic) & 1574 & - \\
\hline$Q_{w}$ (simulation) & 1533 & 1540 \\
\hline$Q_{w}$ (measured) & 1252 & 1305 \\
\hline \hline$Q_{e}$ (simulation) & $1400 / 1370$ & 583 \\
\hline$Q_{e}$ (measured) & 1048 & 571 \\
\hline \hline$\beta$ & 1.19 & 2.29 \\
\hline$[R / Q]$ (simulation) & $134 \Omega$ & $110 \Omega$ \\
\hline
\end{tabular}

of angular frequencies $\omega$. This may be compared with the theoretical result for single-mode excitation of a resonator [4],

$$
\tilde{S}_{11}=\frac{2 \beta}{1+\beta} \cos \psi e^{j \psi}-1 .
$$

The coupling parameter $\beta=Q_{w} / Q_{e}$ is the ratio of wall quality factor to external (or "diffractive") quality factor. Combining Eq. (5) with Slater's theorem, we may infer the electric field profile by means of a bead-pull. In this way have assessed the tune, and $Q_{e}, Q_{w}$, and $\beta$, as listed in Table I. Measured external quality factor was insensitive to the cleaning steps.

Also seen in Table I are the analytic results for a closed pillbox, and the numerical results for the cavity with beam ports obtained from the finite difference code GdfidL [6]. For the analytic comparison, we employed a rectangular pillbox of interior transverse dimensions $a \times b \approx 2.30 \mathrm{~mm} \times 2.44 \mathrm{~mm}$ and transit length $L \approx 0.50 \mathrm{~mm}$. The corresponding resonance angular frequency is given by $\omega_{0}=\beta_{0} c$, where

$$
\beta_{0}=\sqrt{\frac{\pi^{2}}{a^{2}}+\frac{\pi^{2}}{b^{2}}},
$$

with $c \approx 2.9979 \times 10^{8} \mathrm{~m} / \mathrm{s}$ the speed of light. This gives a frequency of $89.6 \mathrm{GHz}$; accounting for the volume change due to the fillets from an 8-mil diameter EDM wire (about $0.8 \%$ ), we calculate the resonance frequency to be $90.1 \mathrm{GHz}$. The measured frequency of the four cavities varied between 90.1 and $90.8 \mathrm{GHz}$, variation attributable to wire machining errors. Theoretical wall quality factor for the closed rectangular pillbox geometry may be expressed as

$$
\frac{1}{Q_{w}}=2 \frac{R_{s}}{Z_{0}} \frac{1}{\beta_{0} L}\left\{1+\frac{2 \pi^{2} L}{\beta_{0}^{2}}\left(\frac{1}{a^{3}}+\frac{1}{b^{3}}\right)\right\},
$$

with $Z_{0} \approx 377 \Omega$ the impedance of free-space, and $R_{s} \approx 79$ $\mathrm{m} \Omega$ the theoretical surface resistance of a smooth OFE copper surface at the operating frequency. This corresponds to wall quality factor of $1.57 \times 10^{3}$.

The external quality factor, $Q_{e}$, may be calculated by two methods. One is a simple estimation from power transmission through the coupling iris, obtained from HFSS simulation [7]. With a rectangular iris of width $0.83 \mathrm{~mm}$, height $0.5 \mathrm{~mm}$ and thickness $0.25 \mathrm{~mm}$, we find $Q_{e}=1400$. 


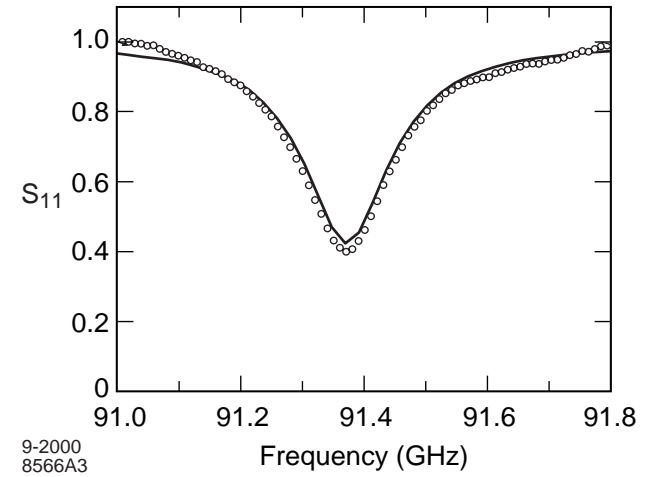

Fig. 3. Reflection coefficient versus frequency, from measurement, and numerical simulation.

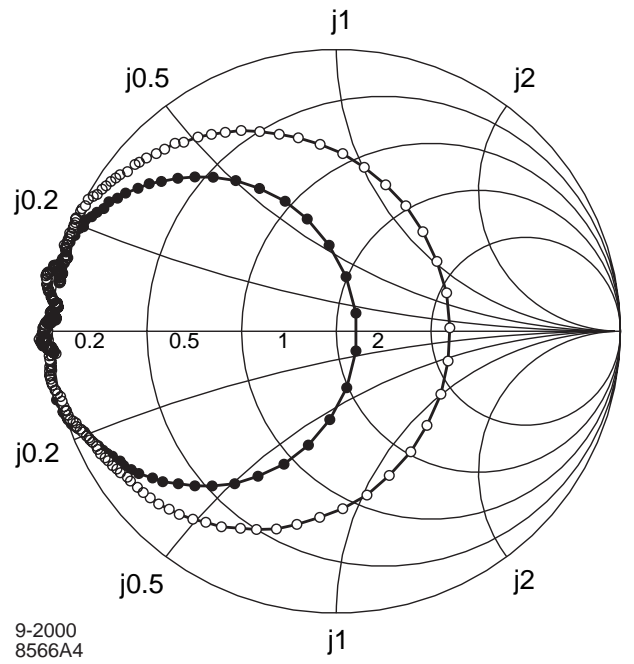

Fig. 4. Smith plot of measured $S_{11}$ for the case of no beam port (black) and with a $680 \mu \mathrm{m}$ beam port (white). For both cases, we see that the cavity is overcoupled.

A second method utilizes the frequency domain result of the closed cavity-waveguide system to find the complex resonance frequency of the waveguide loaded cavity [8]. This gives a $Q_{e}$ of 1370 with no beam port. These dimensions correspond to the geometry prior to cutting of the beam tube and the second acid rinse.

The finished assembly, with beam port, had wider iris opening, of $0.92 \mathrm{~mm}$. For this aperture the frequencydomain approach gives $Q_{e} \approx 583$, lowered due to the resulting stronger external coupling. The sensitivity of $Q_{e}$ to the coupling iris width is noteworthy. A $20-\mu \mathrm{m}$ wider iris lowers the $Q_{e}$ by 200 , while a $20-\mu \mathrm{m}$ thinner iris reduces $Q_{e}$ by 170 .

The result for the scattering parameter $S_{11}$ is indicated in Fig. 3, overlaid with the result computed with the time-domain module of $G d f i d L$. The simulation employs a damping decrement based on the theoretical wall quality factor $Q_{w} \approx 1540$. The same result is seen in Smith-Chart form in Fig. 4. There one can also observe the effect of the chemical cleaning in decreasing the external quality factor. The original iris width was $0.87 \mathrm{~mm}$, and this was widened to $0.92 \mathrm{~mm}$ in the course of chemical cleaning. A bead pul-

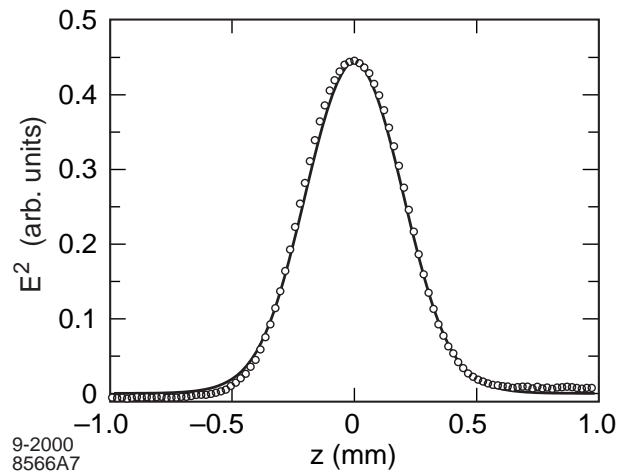

Fig. 5. Result for inferred axial electric field $E_{z}$ from bead-pull measurement, overlaid with theory. The $z$ coordinate is as shown in Fig. 2, with the bead pull along the beam axis.

1 was also performed, with the measurement seen in Fig. 5 , overlaid with the result computed with the frequencydomain module of GdfidL. The dielectric bead was formed by tieing a knot in a length of surgical fiber made of nylon.

The field profile data seen in Fig. 5 permit one to go on to infer peak electric field from cavity voltage, with the result $V_{c} / E_{p k} \approx 6.14 \times 10^{-4} \mathrm{~m}$. Using Eq. (4) one may then infer peak field from power radiated. With $[R / Q] \approx 110 \Omega$ from numerical simulation (GdfidL), and $Q_{e} \approx 571$ from measurement, a figure of $P_{\text {out }} \approx 1 \mathrm{~kW}$ corresponds to a cavity voltage of $7.9 \mathrm{kV}$, and a peak field of $E_{p k} \approx 12.9$ $\mathrm{MV} / \mathrm{m}$.

These bench measurements and simulations indicate that fabrication of, and bench measurement on a high- $Q$ mmwave waveguide-coupled resonator are quite feasible in practice. Results presented here provide the basis for understanding of the cavity interaction with a beam. This cavity has subsequently been installed on a $300 \mathrm{MeV}, 0.5 \mathrm{~A}$ beamline at SLAC, producing peak power in the range of $1 \mathrm{~kW}$, the subject of a later work.

The authors thank G. Caryotakis, J. Huth, O. Millican, D. Shelly, and A. Farvid for their help.

\section{REFERENCES}

[1] D.H. Whittum, "Ultimate gradient in solid-state accelerators", Proceedings of the Advanced Accelerator Concepts Workshop (AIP, to be published), SLAC-PUB-7910.

[2] G. Caryotakis, "High Power Microwave Tubes: In the Laboratory and On-Line", IEEE Trans. Plasma Sci. 22, pp. 683-691, 1994.

[3] G. Caryotakis, "The Klystron: A Microwave Source of Surprising Range and Endurance", Phys. Plasmas 5, pp. 1590-1598, 1998.

[4] D.H. Whittum, "Introduction to Microwave Linacs", in Techniques and Concepts of High-Energy Physics, T. Ferbel, ed. (Kluwer, Amsterdam, 1999) Vol. X, pp. 387-486.

[5] R.H.Siemann, "W-Band vector network analyzer based on an audio lock-in amplifier", SLAC-PUB-7884.

[6] W. Bruns, "GdfidL: A finite difference program for arbitrarily small perturbations in rectangular geometries", IEEE Trans. Magn. 32, pp. 1453-1456, 1996.

[7] "High Frequency Structure Simulator", Ansoft Corp., Four Station Square, Suite 660, Pittsburgh, PA 15219.

[8] Norman M. Kroll and X.E. Lin, "Computer Determinations of the Properties of Waveguide Loaded Cavities", Proceedings of the 1990 Linear Accelerator Conference, LA-12004-C, Carolyn Beckmann, ed. (LANL, Abuquerque, 1990) pp. 238-240. 